\title{
Cefazolin Versus Anti-Staphylococcal Penicillins for the Treatment of Patients with Methicillin- Susceptible Staphylococcus aureus Infection: A Meta- Analysis with Trial Sequential Analysis
}

\author{
John M. Allen (D) - Lolade Bakare - Anthony M. Casapao • \\ Ken Klinker · Lindsey M. Childs-Kean · Ariel F. Pomputius
}

Received: June 14, 2019 / Published online: August 8, 2019

(C) The Author(s) 2019

\section{ABSTRACT}

Introduction: Methicillin-susceptible Staphylococcus aureus (MSSA) is a common cause of infection in humans. Beta-lactam antibiotics are the preferred agents, with anti-staphylococcal penicillins (ASPs) or the first-generation cephalosporin, cefazolin, favored by clinicians. Recent studies comparing the two strategies suggest similar outcomes between the agents. The purpose of this meta-analysis was to explore

Enhanced Digital Features To view enhanced digital features for this article go to https://doi.org/10.6084/ m9.figshare.9009701.

Electronic supplementary material The online version of this article (https://doi.org/10.1007/s40121019-00259-4) contains supplementary material, which is available to authorized users.

J. M. Allen $(\bowtie)$

Department of Pharmacotherapy and Translational Research, University of Florida College of Pharmacy, Orlando, FL, USA

e-mail: john.allen@cop.ufl.edu

J. M. Allen

Department of Internal Medicine, University of Central Florida College of Medicine, Orlando, FL, USA

L. Bakare

University of Florida College of Pharmacy, Orlando, FL, USA differences between cefazolin and ASPs for the treatment of MSSA infections.

Methods: We performed a meta-analysis with trial sequential analysis (TSA) of observational or cohort studies using a random-effects model. Two blinded reviewers independently assessed studies for inclusion, risk of bias, and data extraction. The primary outcome was all-cause mortality. Secondary outcomes included clinical failure, infection recurrence, and antibiotic discontinuation due to adverse events. Subgroup analyses were conducted for the primary outcome by type of ASP, studies with a high percentage of deep-seated infections, and studies of low to moderate risk of bias.

Results: After performing a comprehensive search of the literature, and screening for study inclusion, 19 studies (13,390 patients) were included in the final meta-analysis. Fifteen of the 19 studies (79\%) were judged as having a

\section{A. M. Casapao \\ Department of Pharmacotherapy and Translational Research, University of Florida College of Pharmacy, Jacksonville, FL, USA}

K. Klinker · L. M. Childs-Kean

Department of Pharmacotherapy and Translational Research, University of Florida College of Pharmacy, Gainesville, FL, USA

\section{A. F. Pomputius}

Biomedical and Health Information Services, University of Florida Health Science Center Libraries, Gainesville, FL, USA 
low or moderate risk of bias. Use of cefazolin was associated with lower all-cause mortality [odds ratio (OR) $0.71,95 \%$ confidence interval (CI) $\left.0.56-0.91, p=0.006, I^{2}=28 \%\right]$, clinical failure (OR 0.55 , 95\% CI $0.41-0.74, p<0.001$, $I^{2}=0 \%$, and antibiotic discontinuation due to adverse events (OR 0.25, 95\% CI 0.16-0.39, $\left.p<0.001, I^{2}=23 \%\right)$. Infection recurrence was higher in the cefazolin patients (OR 1.41, 95\% CI 1.04-1.93, $p=0.03, I^{2}=0 \%$ ).

Conclusion: This meta-analysis demonstrated that the use of cefazolin was associated with significant reductions in all-cause mortality, clinical failure, and discontinuation due to adverse events, but was associated with an increased risk of infection recurrence.

Funding: University of Florida Open Access Publishing Fund funded the Rapid Service Fees.

Trial registration: PROSPERO International Prospective Register of Systematic Reviews (study ID: CRD42018106442).

Keywords: Bacteremia; Cefazolin; Infection; Meta-analysis; Penicillins; Staphylococcus aureus

\section{INTRODUCTION}

Staphylococcus aureus (S. aureus) is a gram-positive bacterium that is a part of normal human skin flora. $S$. aureus is the causative pathogen for a wide array of clinically significant infections, ranging from skin and soft tissue infections to more severe infections including endocarditis, bacteremia, pneumonia, and osteomyelitis. $S$. aureus infections are typically broken down into two categories based on their susceptibility profile, methicillin-susceptible (MSSA) or methicillin-resistant (MRSA), with MRSA being associated with higher mortality rates [1] and fewer treatment options. Despite these data, MSSA is still a clinical concern as it still accounts for $>50 \%$ of all $S$. aureus bacteremias, with reported mortality rates of $15-30 \%$, depending on patient comorbidities [2]. Selection of an appropriate treatment regimen is an optimal component of ensuring optimal outcomes in patients with MSSA infections.

Historically, beta-lactams have been the preferred agents for the treatment of MSSA infections, owing to being more efficacious than other agents such as vancomycin, reducing overall mortality, and preventing infection recurrence [3]. Within the beta-lactam drug class, anti-staphylococcal penicillins (ASPs) such as nafcillin and oxacillin are often utilized as first-line agents to treat MSSA infections. Concerns with the frequency of dosing, relatively high acquisition cost, and patient tolerance associated with ASPs have led to an interest in suitable alternatives. Cefazolin is a first-generation cephalosporin that has in vitro activity against MSSA and has a favorable pharmacokinetic profile allowing for less frequent dosing compared with ASPs. However, this enthusiasm is tempered by in vitro data that suggest that an inoculum effect can occur, resulting in higher rates of antibiotic failure compared with ASPs for the treatment of MSSA infections with high bacterial inoculum [4]. However, despite these in vitro data, the impact of the inoculum effect on clinically relevant outcomes is controversial [5]. Additionally, the data are unclear on whether there is a difference between ASPs and cefazolin for the treatment of MSSA infections. The purpose of this metaanalysis is to determine which agent is more effective at reducing mortality and infection recurrence. Additionally, the study will evaluate whether there is any difference related to adverse events between the two treatment strategies.

\section{METHODS}

This meta-analysis was performed based on the methodology recommended by the Cochrane Collaboration and adhered to recommendations according to the Preferred Reporting Items for Systemic Reviews and Meta-analyses statement [6]. The study protocol was submitted and registered with the PROSPERO International Prospective Register of Systematic Reviews (study ID: CRD42018106442) before the start of the literature search. No specific funding sources were utilized to complete this study. This article is based on previously conducted studies and does not contain any studies with human participants or animals performed by any of the 
authors. Our hypothesis was that, compared with ASPs, cefazolin would be associated with reduced mortality and have superior safety outcomes [7].

\section{Eligibility Criteria}

We sought to include all randomized controlled trials utilizing a parallel study design, or observational studies, including cohort and casecontrol studies comparing the use of ASPs with cefazolin. We sought to include only studies in which patients received MSSA treatment with cefazolin or ASP for at least $72 \mathrm{~h}$. No restrictions were imposed based on publication status. We excluded studies conducted in pediatric patients, studies that did not report mortality as an outcome, studies that used antibiotics other than cefazolin or ASPs, studies that included bacteria other than MSSA, and animal and in vitro studies.

\section{Search Strategy}

A comprehensive population, intervention, comparator, and outcome question was created using applicable medical subject headings $(\mathrm{MeSH})$ and individual substances to answer the question, "Does cefazolin reduce all-cause mortality compared with antistaphylococcal penicillins for the management of MSSA infections?" The initial search was conducted by a medical librarian (AFP) across PubMed/Medline, Web of Science, BIOSIS, and Cochrane CENTRAL Register of Controlled Trials. Gray literature was searched using ClinicalTrials.gov, Agency for Healthcare Research and Quality (AHRQ), and infectious diseases professional organizations. In each database, search strategies used controlled vocabulary, if applicable, as well as a variation of keywords related to cefazolin, staphylococcal infections, and antistaphylococcal penicillin. Additionally, relevant conference proceedings and abstracts identified in the search were included if no resulting peerreviewed article was found. A secondary search of Google Scholar was also performed to identify other relevant articles. All references, recent systematic reviews, and guidelines were hand- searched for trials and citations not included from the initial search. Search results were not limited by language or date. The literature search was initially conducted in September 2018 and re-run in June 2019. Full search strategies are available in the supplementary appendix.

\section{Study Selection}

Two investigators (JMA, AMC) independently screened each article for eligibility and inclusion using an electronic spreadsheet (Microsoft Corp., Redmond, WA). Disagreements were resolved by adjudication between the investigators until consensus was achieved.

\section{Data Extraction}

Electronic data collection forms were developed to assess primary and secondary outcomes. Data were also collected on inclusion/exclusion criteria for each study, patient demographics (i.e., age, gender, deep-seated infection, ICU admission at baseline, history of IV drug abuse, baseline need for hemodialysis), year of publication, and type of intervention (drug, dose, duration). Deep-seated infections were defined as osteomyelitis, endocarditis, or pneumonia. Using an electronic data extraction tool, two investigators (JMA, LB) independently extracted data from each study. Disagreements were resolved by adjudication until consensus was achieved.

\section{Risk of Bias Assessment}

Individual risk of bias at the study level was assessed independently by two investigators (JMA, LB) using the Risk of Bias in Non-randomized Studies of Interventions (ROBINS-I) for non-randomized studies [8]. Disagreements were resolved by adjudication between the investigators until consensus was achieved. A priori sensitivity analyses were performed to test the effect of only including studies with low or moderate risk of bias. 


\section{Statistical Analysis}

The primary study outcome was all-cause mortality, reported as odds ratio (OR), and 95\% confidence intervals (95\% CI). Secondary outcomes included clinical failure, infection recurrence, and antibiotic discontinuation due to adverse events. Subgroup analyses were conducted for the primary outcome by type of ASP used, studies of low to moderate risk of bias, studies utilizing at least $4 \mathrm{~g}$ /day of cefazolin, and studies with a high percentage of deepseated infections. Studies with a high percentage of deep-seated infections were defined as having $>25 \%$ of cefazolin patients with deepseated infections (i.e., pneumonia, osteomyelitis, and endocarditis) [9]. Due to the anticipated variability in patient populations, risk factors, and interventions across included studies, all outcomes were analyzed using the MantelHaenszel random-effects model. Heterogeneity among trials was quantified by using the inconsistency factor $\left(I^{2}\right)$. All $p$ values $\leq 0.05$ were considered statistically significant. Statistical analysis was conducted using the Cochrane Review Manager v.5.3 (Cochrane, London, UK) and the Trial Sequential Analysis (TSA) software published by the Copenhagen Trial Unit, Centre for Clinical Intervention Research (available at http://www.ctu.dk/tsa/ index.html). TSA is a methodology that combines an information size calculation (i.e., cumulated sample sizes of all included trials) for a meta-analysis with the threshold of statistical significance. A sensitivity analysis using TSA was performed to confirm the findings of the meta-analysis. Furthermore, before the required information size is reached, TSA constructs monitoring boundaries to determine when an estimated effect is so convincingly large (or small) that the conclusions are unlikely to change with more evidence, which is analogous to an interim analysis of a single randomized controlled trial. The use of TSA to validate metaanalysis results has previously been described [10]. An additional sensitivity analysis to assess the robustness of the results compared with unmeasured confounding using the $E$ value methodology was performed $[11,12]$. The $E$ value estimates what the relative risk would have to be for any unmeasured confounder to overcome the observed association of cefazolin with mortality in this study.

\section{RESULTS}

\section{Study Characteristics}

The initial study search yielded 2211 studies. After de-duplication, 979 studies were included in the initial screening process. After a review of titles and abstracts, and subsequent full-text review, 961 studies were excluded. A subsequent study search yielded an additional study that was not included in the original search, leaving 19 studies in the final analysis (13,390 patients) [13-31]. There was one prospective, observational study included in the final analysis, while the remaining studies were retrospective study designs. The study inclusion flow diagram is included in the study supplemental information. Among the included studies, four had a serious risk of bias (21\%), seven had a moderate risk of bias (37\%), and eight had a low risk of bias $(42 \%)$. The risk of bias for each included study is described in the supplemental study information. Of the included studies, 12 utilized nafcillin as the comparator ASP. Other ASPs included in the analysis were cloxacillin $(n=3)$, oxacillin $(n=2)$, floxacillin $(n=1)$, and nafcillin/oxacillin $(n=1)$. Nineteen of the studies included only patients with positive blood cultures for MSSA. The most-reported follow-up period for mortality was 30 day, with 11 studies reporting this as a study outcome. Other periods reported include 90-day $(n=4)$, in hospital $(n=3)$, and 28-day $(n=1)$. Among all studies in which specific sources of infection were reported, 3343/12,649 (26.4\%) patients had a deep-seated infection (cefazolin, 660/2819, 23.4\%; ASPs, 2683/9830, 27.3\%). A similar number of patients in both groups required ICU admission at study baseline (cefazolin, 395/2454, 16.1\%; ASPs, 1407/8902, $15.8 \%)$. Patients with a history of IV drug abuse were similar between cefazolin and ASPs (cefazolin, 158/1472, 10.7\%; ASPs, 288/2336, $12.3 \%)$. Other specific study characteristics are summarized in Table 1. 


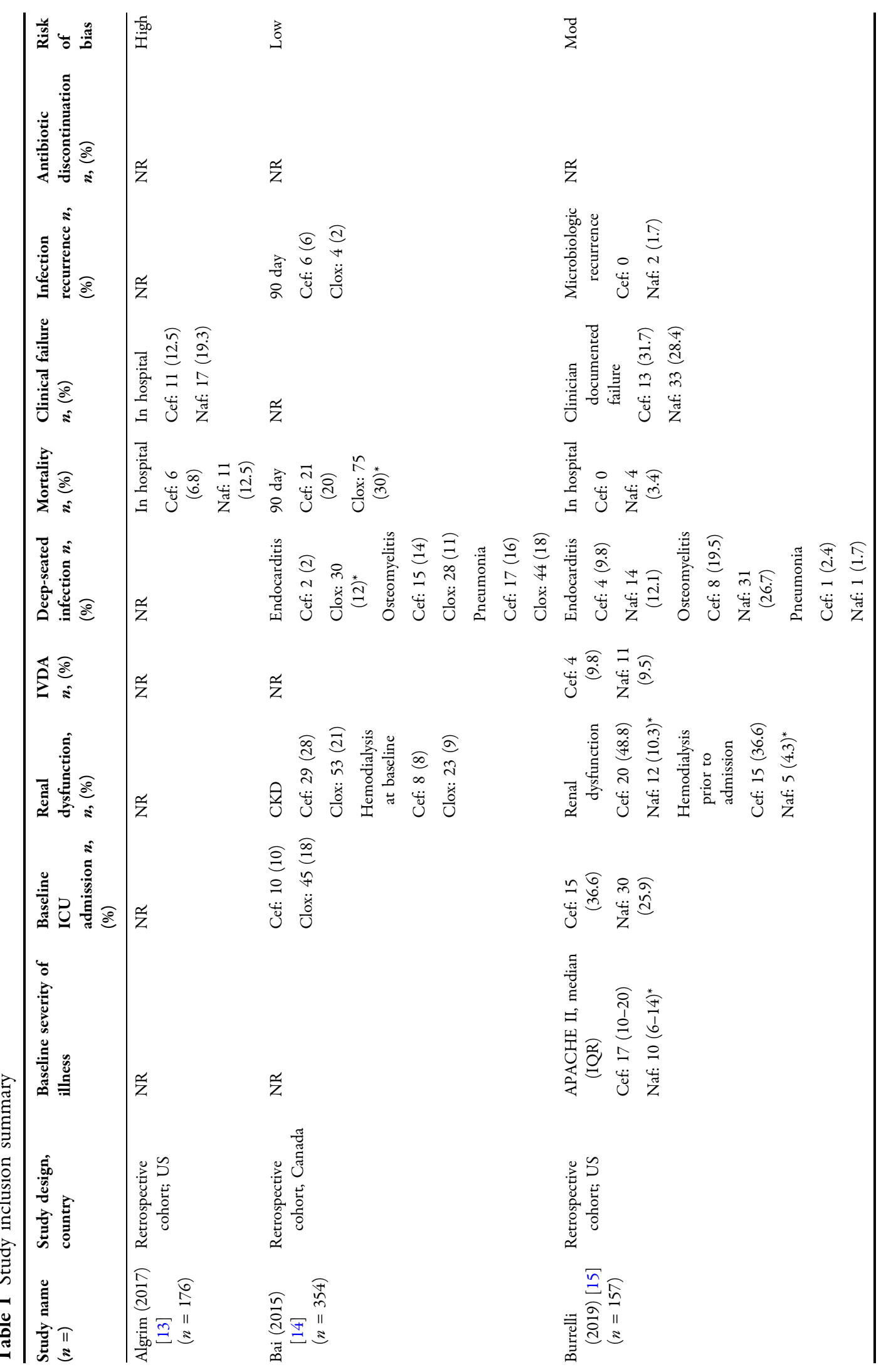




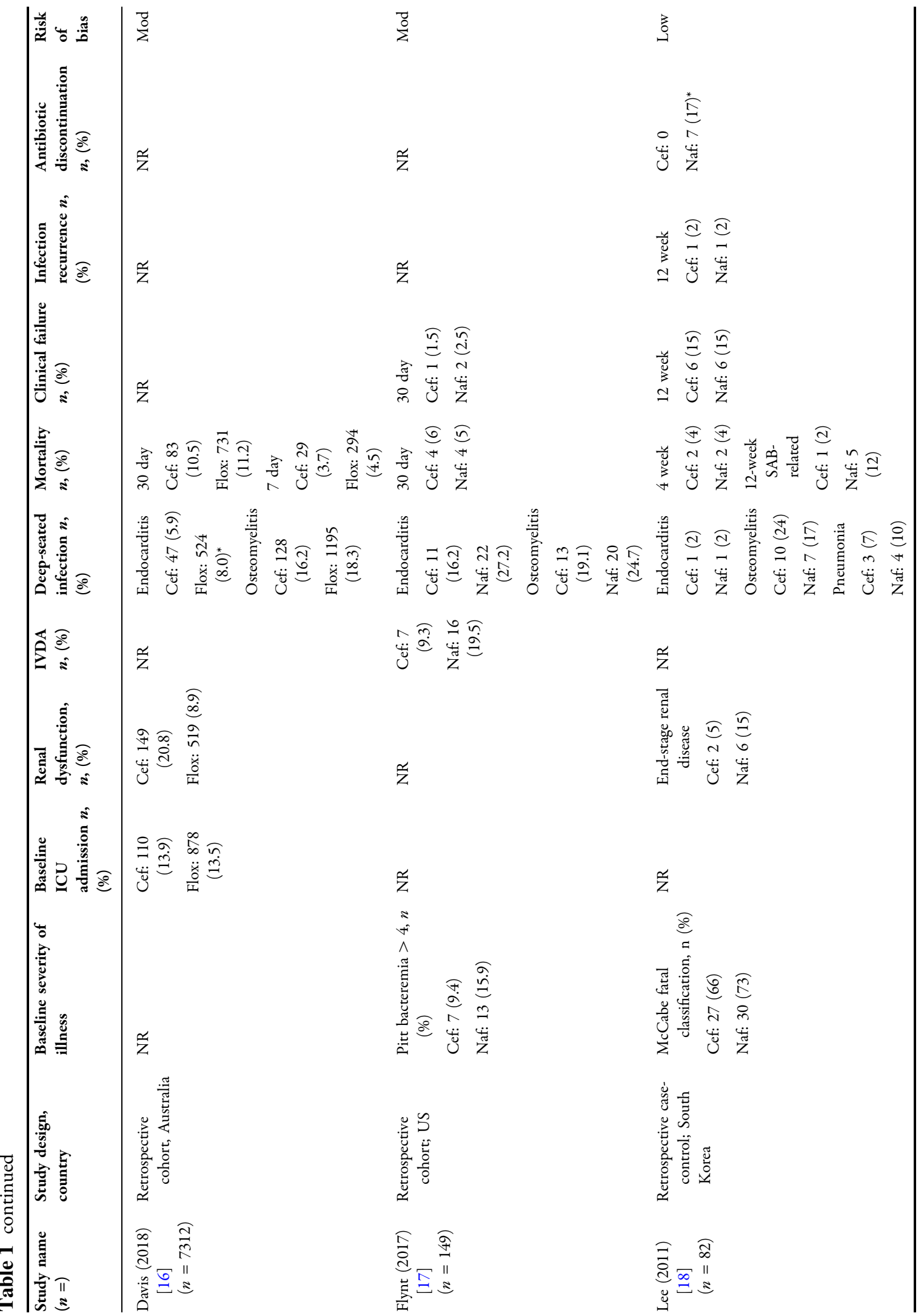




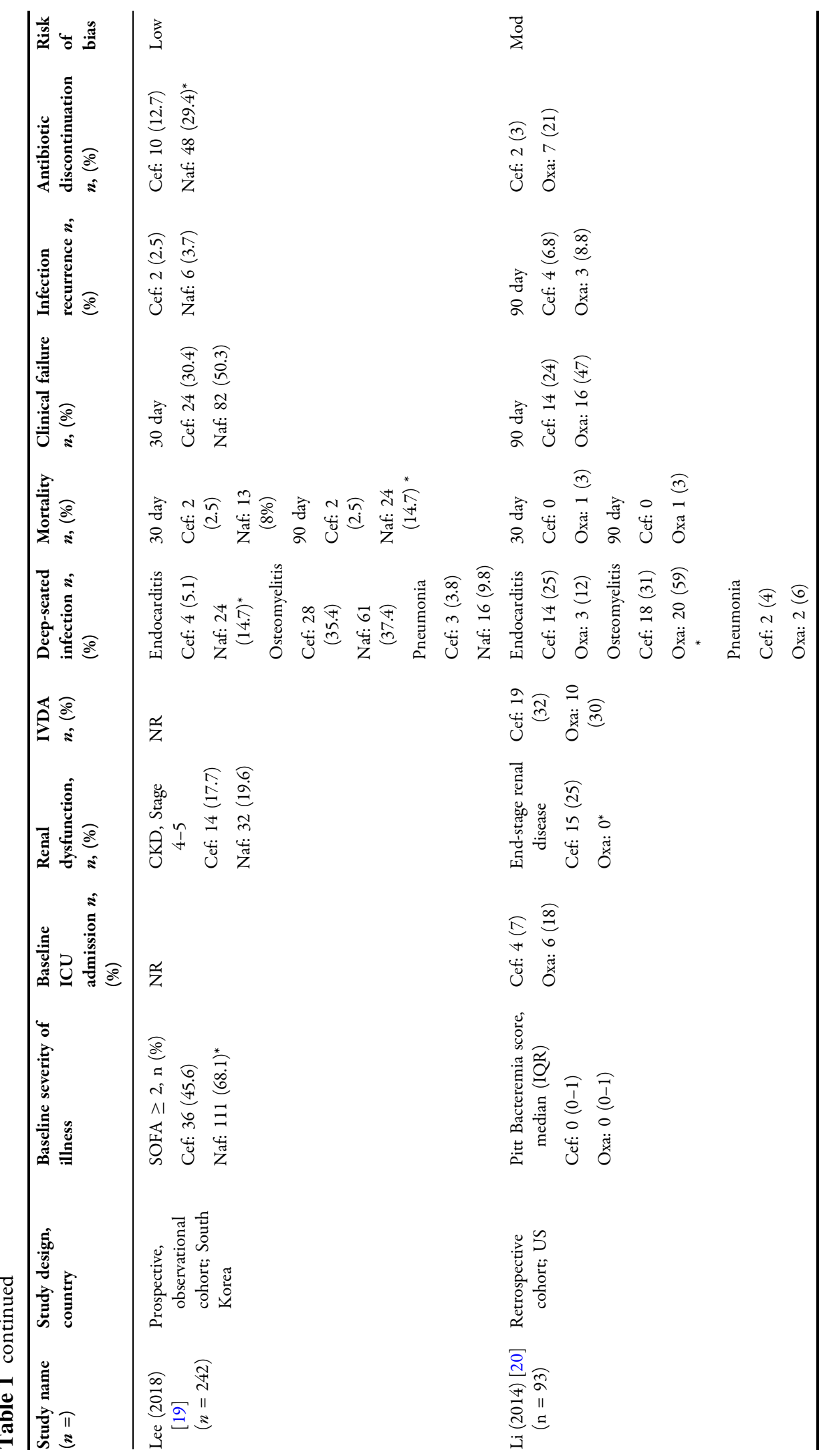




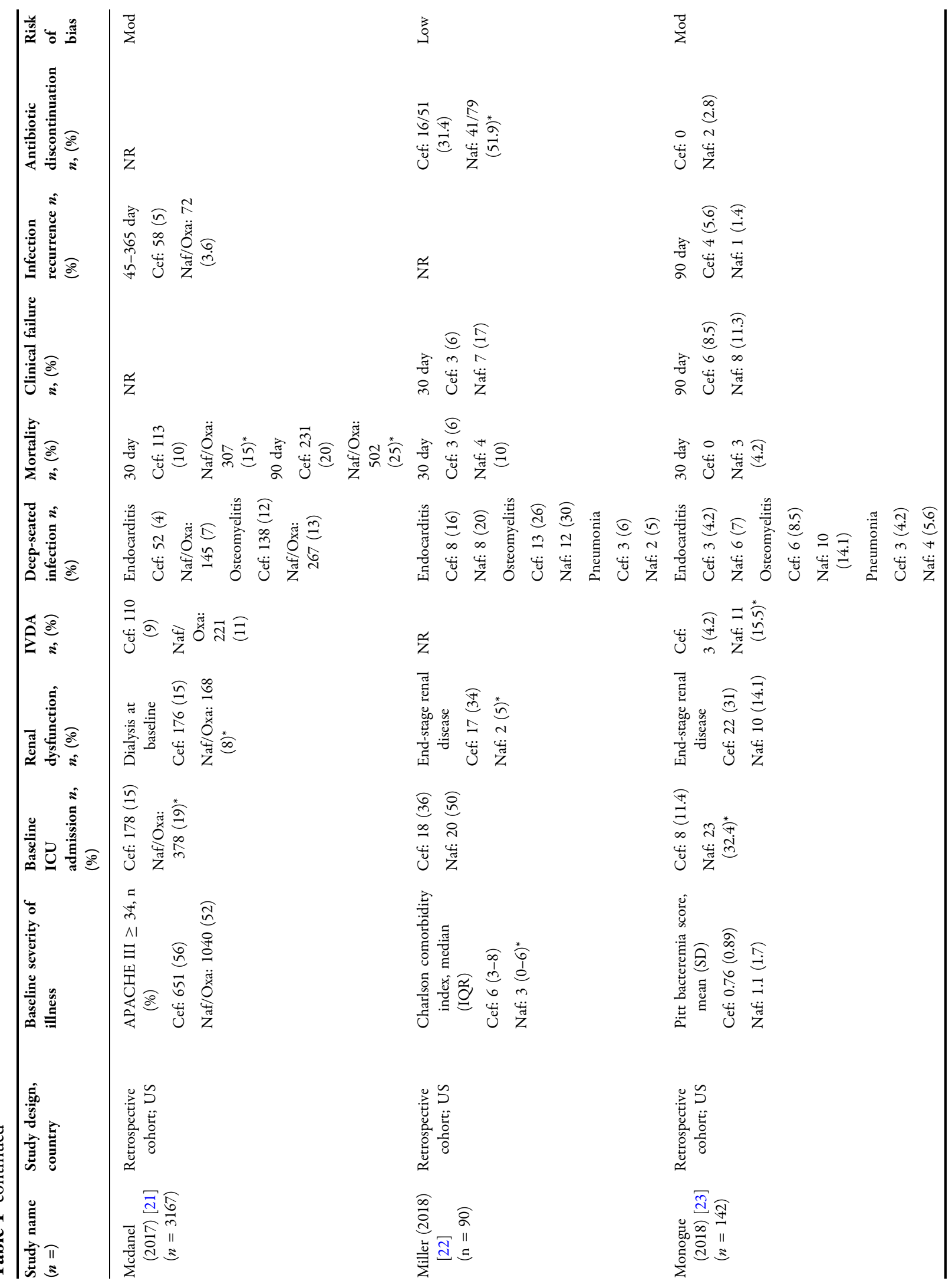




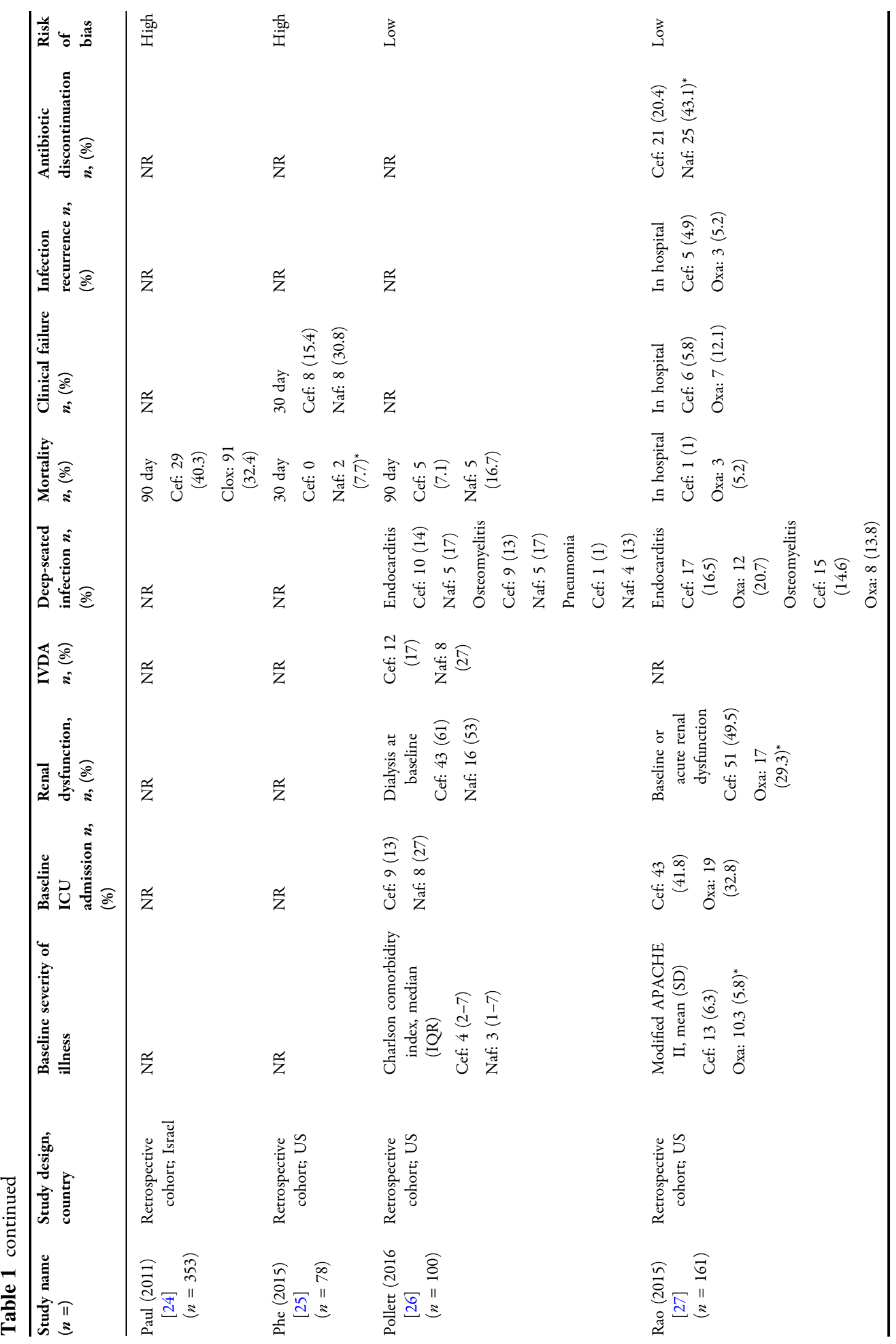




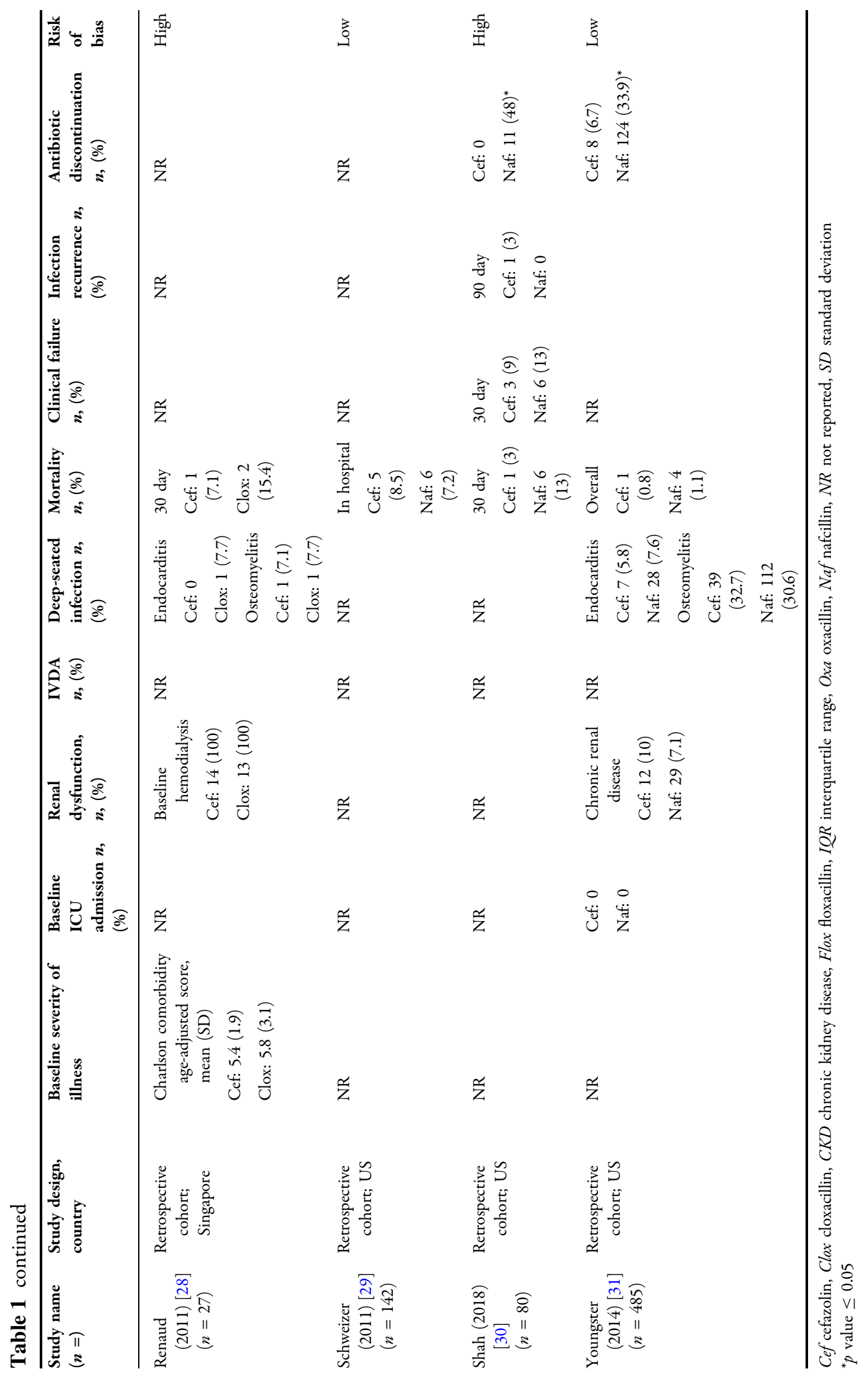




\section{Primary Outcome}

When all studies were included, the all-cause mortality was $12.8 \%(394 / 3081)$ in the cefazolin group and $14.4 \%(1483 / 10309)$ in the ASP group. The use of cefazolin was associated with lower all-cause mortality compared with ASPs (OR $0.71 ; \quad 95 \% \quad$ CI $0.56-0.91 ; \quad p=0.006$; $\left.I^{2}=28 \%\right)$. Among a priori subgroup analysis, cefazolin was also associated with lower allcause mortality compared with ASPs in studies with low/moderate risk of bias (OR 0.75; 95\% CI $\left.0.59-0.96 ; p=0.02 ; I^{2}=32 \%\right)$, studies that used nafcillin (OR 0.46; 95\% CI 0.29-0.74; $p=0.001$; $I^{2}=0 \%$, and studies with a high percentage of deep-seated infections (OR 0.48; 95\% CI $\left.0.32-0.73 ; p=0.0005 ; I^{2}=0 \%\right)$. In studies that utilized at least $4 \mathrm{~g} /$ day of cefazolin, there were no differences observed in all-cause mortality between cefazolin and ASPs (OR 0.55; 95\% CI $\left.0.24-1.24 ; p=0.15 ; I^{2}=0 \%\right)$. The results were unaffected when sensitivity analysis was performed evaluating the impact of the two largest included trials. Figure 1 summarizes all-cause mortality for all included studies and multiple a priori subgroup analyses. Sensitivity analysis of the primary outcome was also performed based on studies with low risk of bias (Supplement). To assess the validity of the meta-analysis results, TSA was performed for all-cause mortality, using various power and zero-event handling assumptions. TSA confirmed the meta-analysis results for most assumed event rates, powering, and zero-event analyses (Supplement). The $E$ value (relative risk) for the point estimate for all-cause mortality was 2.17.

\section{Secondary Outcomes}

Eleven studies reported results on clinical failure with cefazolin use being associated with less clinical failure compared with ASPs (OR 0.55; 95\% CI 0.41-0.71; $p<0.0001 ; I^{2}=0 \%$ ) (Supplement). Upon completion of TSA, significance was maintained (OR 0.55; 95\% CI 0.32-0.97). Nine studies had extractable data on infection recurrence, with cefazolin-treated patients having high risk of infection recurrence compared with ASPs (OR 1.41; 95\% CI 1.04-1.93; $p=0.03$;
$\left.I^{2}=0 \%\right)$ (Supplement), and TSA confirmed these results (OR 1.41; 95\% CI 1.02-1.96). Eight studies reported on antibiotic discontinuation due to adverse events occurring less frequently in cefazolin-treated patients compared with ASPs (OR 0.25; 95\% CI 0.16-0.39; $p<0.001$; $\left.I^{2}=23 \%\right)$ (Supplement). TSA confirmed the meta-analysis results (OR $0.26 ; 95 \%$ CI 0.11-0.62).

\section{DISCUSSION}

Our study results suggest that cefazolin is associated with a small benefit in all-cause mortality compared with ASPs. Additionally, in our a priori subgroup analysis of all-cause mortality, cefazolin continued to be associated with less mortality across multiple subgroups, including studies with low/moderate risk of bias, studies that used nafcillin as the ASP comparator, and studies with high rates of deep-seated infections. One particular subgroup analysis that warrants additional discussion is deep-seated infections. Deep-seated infections such as endocarditis, osteomyelitis, and pneumonia can be notoriously difficult to treat, often require prolonged courses of therapy, and are associated with a higher degree of treatment failure. Previous literature questioned the utility of cefazolin for deep-seated MSSA infections because of an inoculum effect [32]. In our a priori subgroup analysis of studies with a high percentage of deep-seated infections, cefazolin outperformed ASPs in all-cause mortality, suggesting that it could be a reasonable therapeutic option in this patient population. TSA also confirmed the meta-analysis result for all-cause mortality across multiple power and zero-constant assumptions. Cefazolin also was associated with reduced risk of clinical failure and antibiotic discontinuation due to adverse drug events compared with ASPs. The substantially lower risk of clinical failure and antibiotic discontinuation due to adverse drug events with cefazolin compared with ASPs indicated that patients with MSSA infections were more likely to complete therapy on cefazolin than to switch to another agent. Combined with the observed mortality benefit, these findings appear to imply that cefazolin may be the 


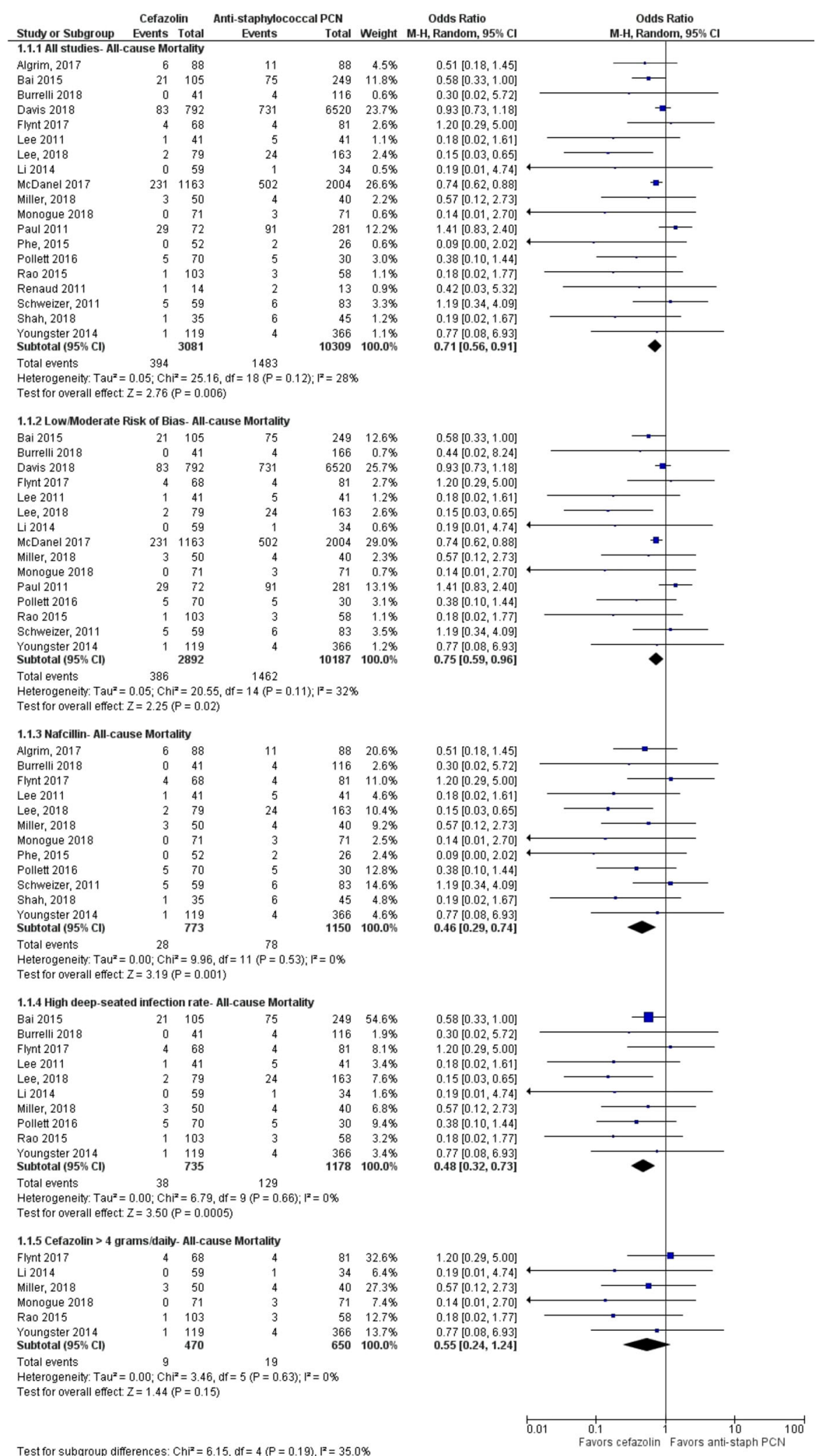

Fig. 1 All-cause mortality 
preferred option for the treatment of MSSA infections. However, these results should be interpreted with trepidation because of the lack of randomization and retrospective study design of all of the studies included in our analysis. Additionally, 18 of the 19 studies included only patients with MSSA infection complicated by bacteremia. The inclusion of positive MSSA blood cultures as an inclusion criterion reduces potential heterogeneity in the patient population compared with MSSA infections without concurrent bacteremia. However, this limited our ability to detect any differences in treatment effects among patients with MSSA infections complicated with bacteremia compared with MSSA infections without concurrent bacteremia. It is unclear whether cefazolin has benefits compared with ASPs in patients without concurrent bacteremia.

Our study results are consistent with previous meta-analyses completed on this topic. Weis and colleagues completed a meta-analysis of 14 studies ( $n=12,859)$, which showed cefazolin use was associated with lower 30-day mortality and less nephrotoxicity compared with ASPs. No difference was noted in treatment failure or relapse rates [33]. Bidell and colleagues completed a meta-analysis of seven studies $(n=4391$ patients) comparing cefazolin with ASP, which suggested cefazolin was associated with a lower risk of 90-day mortality risk compared with ASPs, but no difference in clinical failure [34]. Shi and colleagues completed a meta-analysis of ten studies ( $n=4779$ patients), which showed cefazolin use was associated with reduced all-cause mortality and less risk of antibiotic discontinuation due to adverse drug events compared with ASPs [35]. Lee and colleagues published a metaanalysis of nine studies ( $n=4442$ patients) in acutely ill patients that showed reduced mortality and lower risk of treatment failure with cefazolin compared with ASPs [36]. Our metaanalysis of 19 studies encompassing over 13,000 patients is the largest meta-analysis to date comparing cefazolin to ASPs for the treatment of MSSA infection and is concordant with the previous studies. Inclusion of a larger study [16] published in 2018, which has not previously been included in multiple meta-analyses [34-36], and inclusion of gray literature explains the increased number of patients in our study. Additionally, our study is the first to apply TSA to confirm the meta-analysis results for cefazolin versus ASPs. Most meta-analyses do not have sufficient statistical power to detect or refute intervention effects. When the number of participants and the corresponding number of trials in a meta-analysis are insufficient, the use of the traditional $95 \%$ confidence interval or the 5\% statistical significance threshold will lead to false conclusions (both type I and type II errors) [9]. Application of TSA to meta-analysis results allows for better interpretation of meta-analysis with small numbers of participants and included studies. In our study, TSA confirmed all of the observed results from the traditional meta-analysis. The finding that TSA confirmed all of our significant study results suggests that, based on the available evidence, cefazolin use is associated with better outcomes compared with ASPs. Additionally, we performed a sensitivity analysis for confounding variables for the primary outcome using the $E$ value methodology. The calculated $E$ value was 2.17, meaning that residual confounding could explain the observed association if an unmeasured covariate exists with a relative risk association at least as large as 2.17 with both ASP use and mortality. However, our meta-analysis is not without limitations. As previously noted, all of the included studies had an observational, retrospective study design, which alone introduces the risk of bias, compared with a prospective, randomized trial design. Additionally, 11 of the 19 included studies had a moderate or serious risk of bias according to the ROBINS-I assessment tool used to identify risk of bias in non-randomized trials. However, sensitivity analysis including trials solely with a low risk of bias, and those with low/moderate risk of bias studies did not affect the results of the primary outcome. An additional limitation is that none of the included studies evaluated the potential presence or influence of the cefazolin inoculum effect among MSSA isolates. Inconsistent study definitions across studies also were observed. Future studies on this topic should have a prospective study design, evaluate for the presence of a cefazolin inoculum effect, and account for flaws commonly noted in retrospective and 
observational studies by including proper randomization of study participants.

\section{CONCLUSION}

The results of our meta-analysis, the largest that has been completed on this topic to date, suggest that cefazolin use is associated with a small benefit in all-cause mortality, including in studies with a high percentage of deep-seated infections such as endocarditis, osteomyelitis, and pneumonia. Cefazolin was also associated with reduced clinical failure and was better tolerated compared with ASPs for the treatment of patients with MSSA infections. These findings were derived from retrospective, observational studies, which weakens the study findings. However, TSA confirmed the benefits of cefazolin compared with ASPs. Further investigation should be undertaken to evaluate whether cefazolin is associated with an increase in infection recurrence in patients with MSSA infections compared with ASPs. Pragmatically, it would appear reasonable to avoid using cefazolin in patient scenarios with a high-risk of infection recurrence until further evidence is available (e.g., MSSA endocarditis without the possibility of surgical management, prosthetic joint infection without the possibility of hardware removal). Due to its favorable pharmacokinetic profile requiring less frequent dosing, and associated positive clinical outcomes, cefazolin appears to be a reasonable alternative to ASPs for the management of MSSA infections. Further research is needed to evaluate for patient-specific factors to guide antibiotic selection in patients with MSSA infections.

\section{ACKNOWLEDGEMENTS}

Funding. University of Florida Open Access Publishing Fund funded the Rapid Service Fees. No funding sources were utilized to complete this study.

Authorship. All named authors meet the International Committee of Medical Journal
Editors (ICMJE) criteria for authorship for this article, take responsibility for the integrity of the work as a whole, and have given their approval for this version to be published.

Disclosures. At the time of this study, Ken Klinker was employed by the College of Pharmacy, University of Florida (Gainesville, FL). Ken Klinker currently works for Merck \& Co., Inc. (Kenilworth, NJ). John M. Allen, Lolade Bakare, Anthony M. Casapao, Lindsey M. Childs-Kean, and Ariel F. Pomputius have nothing to disclose.

Compliance with Ethics Guidelines. This article is based on previously conducted studies and does not contain any studies with human participants or animals performed by any of the authors

Open Access. This article is distributed under the terms of the Creative Commons Attribution-NonCommercial 4.0 International License (http://creativecommons.org/licenses/ by-nc/4.0/), which permits any noncommercial use, distribution, and reproduction in any medium, provided you give appropriate credit to the original author(s) and the source, provide a link to the Creative Commons license, and indicate if changes were made.

\section{REFERENCES}

1. Hanberger H, Walther S, Leone M, Barie PS, Rello J, Lipman J, et al. Increased mortality associated with methicillin-resistant Staphylococcus aureus (MRSA) infection in the intensive care unit: results from the EPIC II study. Int $\mathrm{J}$ Antimicrob Agents. 2011;38(4):331-5.

2. van Hal SJ, Jensen SO, Vaska VL, Espedido BA, Paterson DL, Gosbell IB. Predictors of mortality in Staphylococcus aureus bacteremia. Clin Microbiol Rev. 2012;25(2):362-86.

3. McDanel JS, Perencevich EN, Diekema DJ, Herwaldt LA, Smith TC, Chrischilles EA, et al. Comparative effectiveness of beta-lactams versus vancomycin for treatment of methicillin-susceptible Staphylococcus aureus bloodstream infections among 122 hospitals. Clin Infect Dis. 2015;61(3):361-7. 
4. Nannini EC, Singh KV, Murray BE. Relapse of type A beta-lactamase-producing Staphylococcus aureus native valve endocarditis during cefazolin therapy: revisiting the issue. Clin Infect Dis. 2003;37(9):1194-8.

5. Chong YP, Park SJ, Kim ES, Bang KM, Kim MN, Kim $\mathrm{SH}$, et al. Prevalence of blaZ gene types and the cefazolin inoculum effect among methicillin-susceptible Staphylococcus aureus blood isolates and their association with multilocus sequence types and clinical outcome. Eur J Clin Microbiol Infect Dis. $2015 ; 34(2): 349-55$.

6. Moher D, Liberati A, Tetzlaff J, Altman DG. Preferred reporting items for systematic reviews and meta-analyses: the PRISMA statement. PLoS Med. 2009;6(7):e1000097.

7. Rindone JP, Mellen CK. Meta-analysis of trials comparing cefazolin to antistaphylococcal penicillins in the treatment of methicillin-sensitive Staphylococcus aureus bacteraemia. Br J Clin Pharmacol. 2018;84(6):1258-66.

8. Sterne JA, Hernan MA, Reeves BC, Savovic J, Berkman ND, Viswanathan M, et al. ROBINS-I: a tool for assessing risk of bias in non-randomised studies of interventions. BMJ. 2016;355:i4919.

9. Tong SY, Davis JS, Eichenberger E, Holland TL, Fowler VG Jr. Staphylococcus aureus infections: epidemiology, pathophysiology, clinical manifestations, and management. Clin Microbiol Rev. 2015;28(3):603-61.

10. Wetterslev J, Jakobsen JC, Gluud C. Trial sequential analysis in systematic reviews with meta-analysis. BMC Med Res Methodol. 2017;17(1):39.

11. Mathur MB, Ding P, Riddell CA, VanderWeele TJ. Website and $\mathrm{R}$ package for computing E-values. Epidemiology. 2018;29(5):e45-7.

12. VanderWeele TJ, Ding P. Sensitivity analysis in observational research: introducing the E-value. Ann Intern Med. 2017;167(4):268-74.

13. Algrim A, Twilla J, Samarin M, Cummings C. Comparison of nafcillin and cefazolin for the treatment of methicillin-susceptible Staphylococcus aureus bacteremia. Pharmacotherapy. 2017;37(12):E184. https://doi.org/10.1002/phar. 2052.

14. Bai AD, Showler A, Burry L, Steinberg M, Ricciuto DR, Fernandes T, et al. Comparative effectiveness of cefazolin versus cloxacillin as definitive antibiotic therapy for MSSA bacteraemia: results from a large multicentre cohort study. J Antimicrob Chemother. 2015;70(5):1539-46.
15. Burrelli CC, Broadbent EK, Margulis A, Snyder GM, Gold HS, McCoy C, et al. Does the beta-lactam matter? Nafcillin versus cefazolin for methicillinsusceptible Staphylococcus aureus bloodstream infections. Chemotherapy. 2019;63(6):345-51.

16. Davis JS, Turnidge J, Tong S. A large retrospective cohort study of cefazolin compared with flucloxacillin for methicillin-susceptible Staphylococcus aureus bacteraemia. Int $\mathrm{J}$ Antimicrob Agents. 2018;52(2):297-300.

17. Flynt LK, Kenney RM, Zervos MJ, Davis SL. The safety and economic impact of cefazolin versus nafcillin for the treatment of methicillin-susceptible Staphylococcus aureus bloodstream infections. Infect Dis Ther. 2017;6(2):225-31.

18. Lee S, Choe PG, Song KH, Park SW, Kim HB, Kim NJ, et al. Is cefazolin inferior to nafcillin for treatment of methicillin-susceptible Staphylococcus aureus bacteremia? Antimicrob Agents Chemother. 2011;55(11):5122-6.

19. Lee S, Song KH, Jung SI, Park WB, Lee SH, Kim YS, et al. Comparative outcomes of cefazolin versus nafcillin for methicillin-susceptible Staphylococcus aureus bacteraemia: a prospective multicentre cohort study in Korea. Clin Microbiol Infect. 2018;24(2):152-8.

20. Li J, Echevarria KL, Hughes DW, Cadena JA, Bowling JE, Lewis JS 2nd. Comparison of cefazolin versus oxacillin for treatment of complicated bacteremia caused by methicillin-susceptible Staphylococcus aureus. Antimicrob Agents Chemother. 2014;58(9):5117-24.

21. McDanel JS, Roghmann MC, Perencevich EN, Ohl ME, Goto M, Livorsi DJ, et al. Comparative effectiveness of cefazolin versus nafcillin or oxacillin for treatment of methicillin-susceptible Staphylococcus aureus Infections complicated by bacteremia: a Nationwide Cohort Study. Clin Infect Dis. 2017;65(1):100-6.

22. Miller MA, Fish DN, Barber GR, Barron MA, Goolsby TA, Moine $\mathrm{P}$, et al. A comparison of safety and outcomes with cefazolin versus nafcillin for methicillin-susceptible Staphylococcus aureus bloodstream infections. J Microbiol Immunol Infect. 2018. https://doi.org/10.1016/j.jmii.2018.07.006 (Epub ahead of print).

23. Monogue ML, Ortwine JK, Wei W, Eljaaly K, Bhavan KP. Nafcillin versus cefazolin for the treatment of methicillin-susceptible Staphylococcus aureus bacteremia. J Infect Public Health. 2018;11(5):727-31.

24. Paul M, Zemer-Wassercug N, Talker O, Lishtzinsky $\mathrm{Y}$, Lev B, Samra Z, et al. Are all beta-lactams 
similarly effective in the treatment of methicillinsensitive Staphylococcus aureus bacteraemia? Clin Microbiol Infect. 2011;17(10):1581-6.

25. Phe K, Russo HR. Comparing Clinical Outcomes in Patients Treated With Cefazolin Versus Nafcillin for Methicillin Susceptible Staphylococcus aureus Bacteremia Secondary to High-Inoculum Infections [abstract]. In: Proceedings of the 55th Annual Interscience Conference on Antimicrobial Agents and Chemotherapy. 2015 Sept 17-21; San Diego, CA. ICAAC; 2015. https://www.abstractsonline. com/Plan/ViewAbstract.aspx?sKey=f8c4b522-bc424501-a27f-91fd36a2fa47\&cKey=68acf0cb-7963-452 9-832e-913fd324bd98\&mKey=7a574a80-eab1-4b50 -b343-4695df14907e. Accessed 14 June 2019.

26. Pollett S, Baxi SM, Rutherford GW, Doernberg SB, Bacchetti P, Chambers HF. Cefazolin versus nafcillin for methicillin-sensitive Staphylococcus aureus bloodstream infection in a california tertiary medical center. Antimicrob Agents Chemother. 2016;60(8):4684-9.

27. Rao SN, Rhodes NJ, Lee BJ, Scheetz MH, Hanson AP, Segreti J, et al. Treatment outcomes with cefazolin versus oxacillin for deep-seated methicillin-susceptible Staphylococcus aureus bloodstream infections. Antimicrob Agents Chemother. 2015;59(9):5232-8.

28. Renaud CJ, Lin X, Subramanian S, Fisher DA. Highdose cefazolin on consecutive hemodialysis in anuric patients with Staphylococcal bacteremia. Hemodial Int. 2011;15(1):63-8.

29. Schweizer ML, Furuno JP, Harris AD, Johnson JK, Shardell MD, McGregor JC, et al. Comparative effectiveness of nafcillin or cefazolin versus vancomycin in methicillin-susceptible Staphylococcus aureus bacteremia. BMC Infect Dis. 2011;11:279.

30. Shah MD, Wardlow LC, Stevenson KB, Coe KE, Reed EE. Clinical outcomes with penicillin versus alternative beta-lactams in the treatment of penicillin-susceptible Staphylococcus aureus bacteremia. Pharmacotherapy. 2018. https://doi.org/10.1002/ phar.2124 (Epub ahead of print).

31. Youngster I, Shenoy ES, Hooper DC, Nelson SB. Comparative evaluation of the tolerability of cefazolin and nafcillin for treatment of methicillinsusceptible Staphylococcus aureus infections in the outpatient setting. Clin Infect Dis. 2014;59(3):369-75.

32. Quinn EL, Pohlod D, Madhavan T, Burch K, Fisher $\mathrm{E}$, Cox F. Clinical experiences with cefazolin and other cephalosporins in bacterial endocarditis. J Infect Dis. 1973;128(Suppl):S386-9.

33. Weis S, Kesselmeier M, Davis JS, Morris AM, Lee S, Scherag A, et al. Cefazolin versus anti-staphylococcal penicillins for the treatment of patients with Staphylococcus aureus bacteremia: a systematic review and meta-analysis. Clin Microbiol Infect. 2019;25(7):818-27.

34. Bidell MR, Patel N, O'Donnell JN. Optimal treatment of MSSA bacteraemias: a meta-analysis of cefazolin versus antistaphylococcal penicillins. J Antimicrob Chemother. 2018;73(10):2643-51.

35. Shi C, Xiao Y, Zhang Q, Li Q, Wang F, Wu J, et al. Efficacy and safety of cefazolin versus anti Staphylococcal penicillins for the treatment of methicillinsusceptible Staphylococcus aureus bacteremia: a systematic review and meta-analysis. BMC Infect Dis. 2018;18(1):508.

36. Lee BJ, Wang SK, Constantino-Corpuz JK, Apolinario K, Nadler B, McDanel JS, et al. Cefazolin vs. anti-staphylococcal penicillins for treatment of methicillin-susceptible Staphylococcus aureus bloodstream infections in acutely ill adult patients: Results of a systematic review and meta-analysis. Int J Antimicrob Agents. 2019;53(3):225-33. 